\title{
Editorial
}

\section{Stochastic Optimization: Theory and Applications}

\author{
Bin Wang, ${ }^{1}$ Lotfollah Najjar, ${ }^{2}$ Neal N. Xiong, ${ }^{3}$ and Rung Ching Chen ${ }^{4}$ \\ ${ }^{1}$ Engineering Optimization and Smart Antenna Institute, School of Computer and Communication Engineering, \\ Northeastern University at Qinhuangdao, Hebei, Qinhuangdao 066004, China \\ ${ }^{2}$ Information System Quantitative Analysis, College of Information Science \& Technology (IS\&T), \\ University of Nebraska at Omaha, Omaha, NE 68182, USA \\ ${ }^{3}$ School of Computer Science, Colorado Technical University, 4435 North Chestnut Street, Colorado Springs, CO 80907, USA \\ ${ }^{4}$ College of Informatics Chaoyang University of Technology, 168 Jifong East Road, Wufong District, Taichung City 41349, Taiwan
}

Correspondence should be addressed to Bin Wang; wangbinneu@qq.com

Received 23 October 2013; Accepted 23 October 2013

Copyright (C) 2013 Bin Wang et al. This is an open access article distributed under the Creative Commons Attribution License, which permits unrestricted use, distribution, and reproduction in any medium, provided the original work is properly cited.

As an important branch of applied mathematics, optimization theory, especially stochastic optimization, becomes an important tool for solving multiobjective decision-making problems in random process recently. Many kinds of industrial, biological, engineering, and economic problems can be viewed as stochastic systems, for example, area of communication, gene, signal processing, geography, civil engineering, aerospace, banking, and so forth. Stochastic optimization is suitable to solve the decision-making problems in these stochastic systems.

This special issue includes 16 high-quality peer-reviewed papers that deal with different aspects of stochastic optimization problems. These papers contain some new, novel, and innovative techniques and ideas. We hope that all the papers published in this special issue can stimulate the continuing efforts to understand this field, particularly new stochastic optimization algorithms and new applications in related fields.

In the paper entitled "Qualitative and quantitative integrated modeling for stochastic simulation and optimization," the authors propose a qualitative and quantitative combined modeling specification based on a hierarchical model structure framework. The new modeling approach is based on a hierarchical model structure which includes the meta-meta model, the metamodel, and the high-level model.

In the paper entitled "Estimating time-varying beta of price limits and its applications in China stock market," the authors propose an estimation method of time-varying beta of price limits. It uses China stock market trading data to estimate time-varying beta and researches on systemic risk in China stock market.

In the paper entitled "Doubly constrained robust blind beamforming algorithm," the authors propose doubly constrained robust least squares constant modulus algorithm (LSCMA) to solve the problem of signal steering vector mismatches via the Bayesian method and worst-case performance optimization, which is based on the mismatches between the actual and presumed steering vectors. A theoretical analysis for the proposed algorithm in terms of complexity cost, convergence performance, and SINR performance is presented in this paper.

In the paper entitled "Smoothing techniques and augmented Lagrangian method for recourse problem of twostage stochastic linear programming," the authors apply the smoothing techniques and a fast Newton-Armijo algorithm for solving an unconstrained smooth reformulation of this problem. Computational results and comparisons are given to show the effectiveness and speed of the algorithm.

In the paper entitled "New results on robust stability and stabilization of linear discrete-time stochastic systems with convex polytopic uncertainties," the authors propose new delaydependent mean square robust stability conditions for linear polytopic delay-difference stochastic equations with interval time-varying delays in terms of LMIs. An application to robust stabilization of linear discrete-time stochastic control systems is given in this paper. 
In the paper entitled "Layer-based data aggregation and performance analysis in wireless sensor networks", the authors focus on the minimum-latency data aggregation problem and propose a new efficient scheme for it. The basic idea is to build an aggregation tree by ordering nodes into layers and then use a scheduling algorithm on the basis of the aggregation tree to determine the transmission time slots for all nodes in the network with collision avoiding.

In the paper entitled "Optimal waveform selection for robust target tracking," the authors assume a one-dimension target model which will try to escape the radar detection to degrade the tracking performance and propose a new optimal waveform selection algorithm based on game theory for robust tracking. The optimal parameters for transmitted waveform are finally found by the minimization of the trace of the estimated state error covariance.

In the paper entitled "Adaptive waveform design for multiple radar tasks based on constant modulus constraint," the authors propose a waveform design method which can efficiently synthesize waveforms and provide a trade-off between estimation performance and detection performance and then apply the technique of generating a constant modulus signal with the given Fourier transform magnitude to the waveform. Finally a waveform that has constant modulus property can be obtained.

In the paper entitled "Univex interval-valued mapping with differentiability and its application in nonlinear programming," the authors introduce interval-valued univex functions for differentiable programming problems and derive optimality and duality results for a class of generalized convex optimization problems with interval-valued univex functions.

In the paper entitled "Study on indefinite stochastic linear quadratic optimal control with inequality constraint," the authors study the indefinite stochastic LQ optimal control problem with unequally terminal state constraint, which can be transformed into a hybrid constrained mathematical programming problem and design a dynamic programming algorithm to solve the constrained indefinite stochastic LQ issue.

In the paper entitled "A fast optimization method for reliability and performance of cloud services composition application," the authors propose a fast optimization method for reliability and performance of cloud services composition application based on Universal Generating Function and Genetic Algorithm. The model and algorithm can be applied in online prediction and optimization for reliability and performance of cloud services composition application.

In the paper entitled "Dynamic mean-variance model with borrowing constraint under the constant elasticity of variance process," the authors study a continuous-time dynamic meanvariance portfolio selection problem with the constraint of a higher borrowing rate, in which stock price is governed by a constant elasticity of variance (CEV) process.

In the paper entitled "An improved hybrid genetic algorithm with a new local search procedure," the authors propose a novel, simplified, and efficient HGA with a new individual learning procedure that performs a LS only when the best offspring (solution) in the offspring population is also the best in the current parent population and develop a new LS method based on a three-directional search (TD), which is derivative-free and self-adaptive.

In the paper entitled "Reflected backward stochastic differential equations driven by countable brownian motions," the authors deal with a new class of reflected backward stochastic differential equation driven by countable Brownian motions. The existence and uniqueness of the RBSDEs is obtained via Snell envelope and fixed theorem.

In the paper entitled "Pareto optimal solutions for stochastic dynamic programming problems via Monte Carlo simulation," the authors propose a heuristic algorithm for a class of stochastic discrete-time continuous-variable dynamic programming problems submitted to non-Gaussian disturbances. This new idea is carried out by using Monte Carlo simulations embedded in an approximate algorithm proposed for deterministic dynamic programming problems. The new method is tested in instances of the classical inventory control problem.

In the paper entitled "On iterative learning control for remote control systems with packet loss," the authors propose an ILC for a time-varying system with random packet dropouts. The ILC law adopts an iteration-average operator and a revised learning gain that takes into consideration the probabilities of data-dropout factors.

\section{Acknowledgments}

As guest editors for this special issue, we would like to take this opportunity to thank all the contributions from the authors and reviewers. Bin Wang was supported by the Natural Science Foundation of Hebei Province (no. F2013501075), the Doctoral Scientific Research Foundation of Liaoning Province (no. 20131030), and the Fundamental Research Funds for the Central Universities (no. N110323005).

\author{
Bin Wang \\ Lotfollah Najjar \\ Neal N. Xiong \\ Rung Ching Chen
}




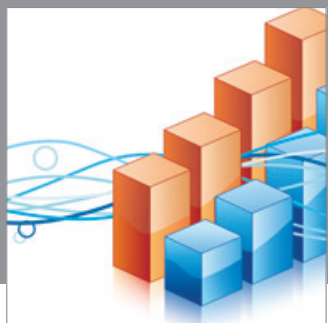

Advances in

Operations Research

mansans

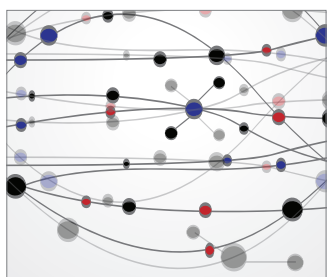

The Scientific World Journal
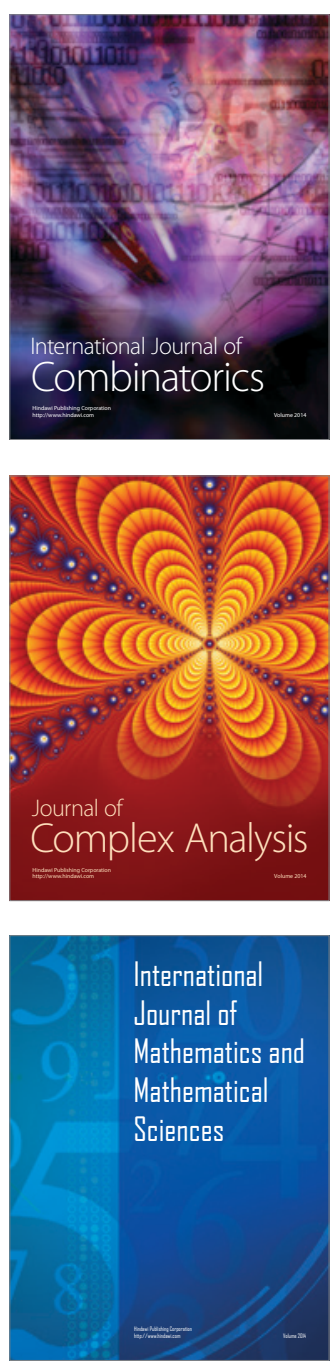
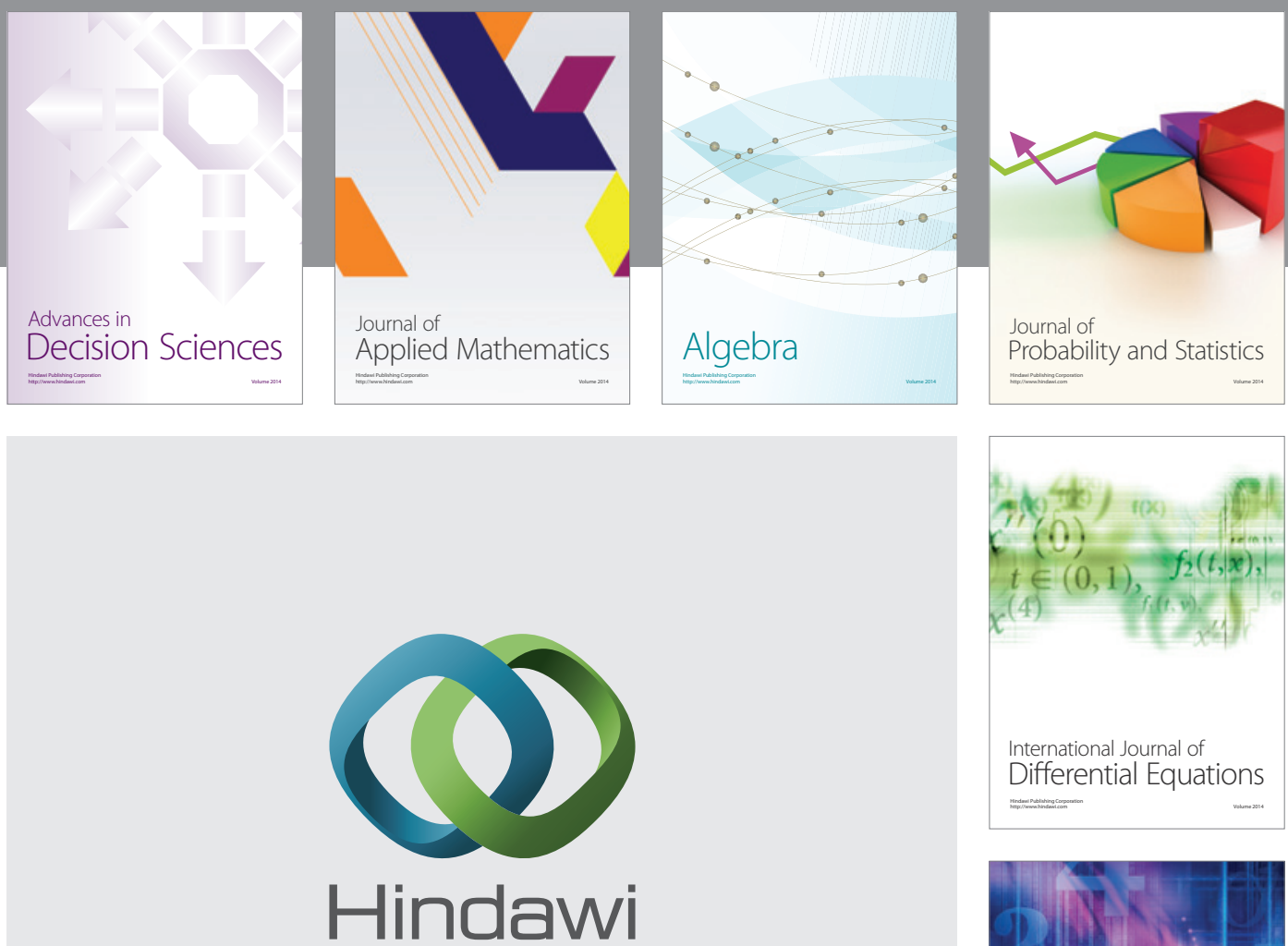

Submit your manuscripts at http://www.hindawi.com
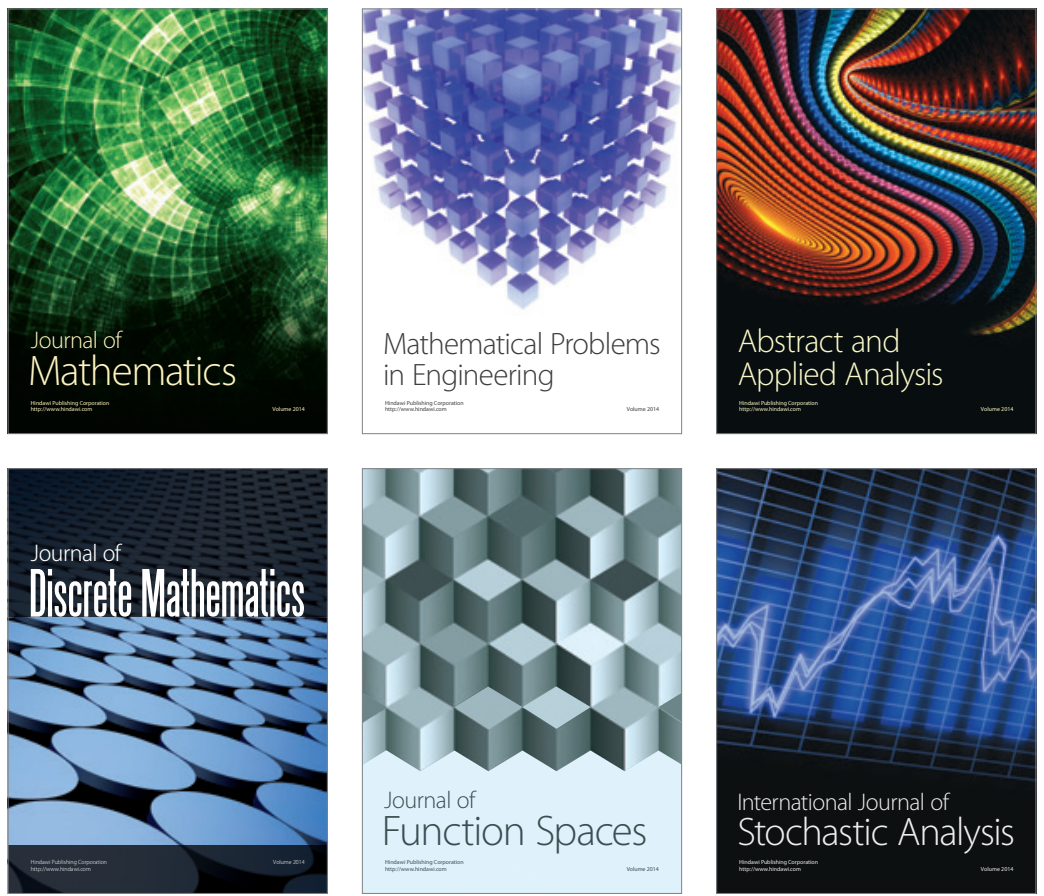

Journal of

Function Spaces

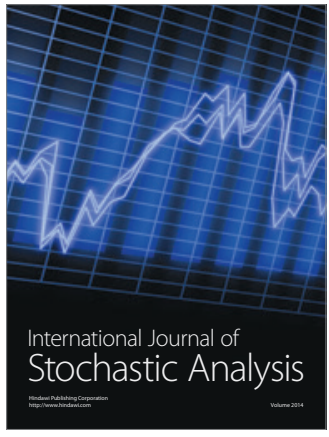

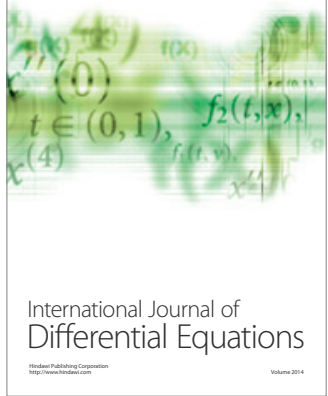
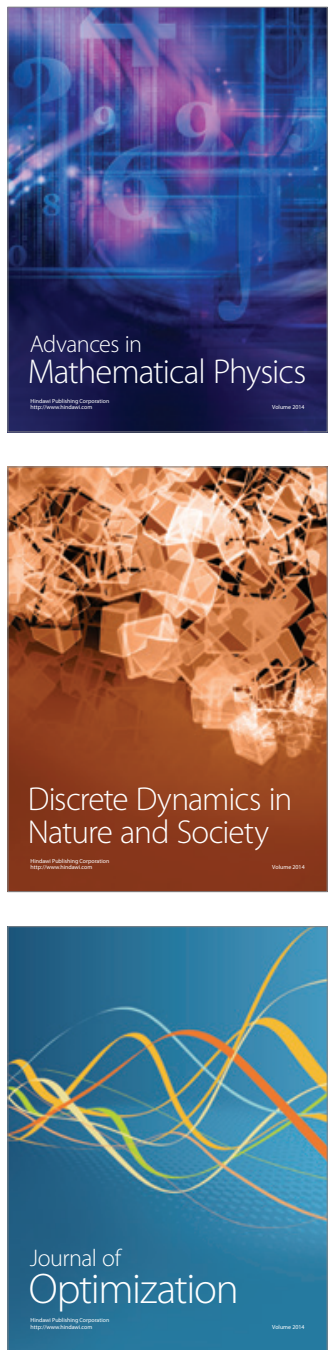